\title{
Production of $\Sigma^{0}$ Hyperons at LHC with ALICE
}

\author{
Alexander Borissov ${ }^{1, *}$ for the ALICE Collaboration \\ ${ }^{1}$ Moscow Institute of Physics and Technology, Dolgoprudny, Russia \\ ${ }^{2}$ National Research Nuclear University MEPhI, Moscow, Russia
}

\begin{abstract}
The first measurements of the transverse momentum $\left(p_{\mathrm{T}}\right)$ spectra and integrated yields of $\Sigma^{0}$ and $\bar{\Sigma}^{0}$ baryons in proton-proton (pp) collisions at $\sqrt{s}=7 \mathrm{TeV}$ are reported. The $\Sigma^{0}\left(\bar{\Sigma}^{0}\right)$ signal is reconstructed via the $\Lambda(\bar{\Lambda})+$ $\gamma$ decay channel by invariant mass analysis. The $\Lambda(\bar{\Lambda})$ baryon is reconstructed by its weak decay into $\mathrm{p}+\pi^{-}\left(\overline{\mathrm{p}}+\pi^{+}\right)$, while the photon is detected exploiting the unique capability of the ALICE detector to measure low energy photons via conversion into $\mathrm{e}^{+} \mathrm{e}^{-}$pairs. A significant disagreement between the data and the predictions from the QCD-inspired Monte Carlo event generator PYTHIA is observed for the $p_{\mathrm{T}}$ spectrum. The yield ratio of $\Sigma^{0}\left(\bar{\Sigma}^{0}\right)$ to $\Lambda$ is shown as a function of $p_{\mathrm{T}}$. Finally, the new data point for the integrated yield ratio of $\Sigma^{0}\left(\bar{\Sigma}^{0}\right)$ to $\Lambda$ from pp collisions at $7 \mathrm{TeV}$ is found to be similar to the results obtained in different elementary collison systems $\left(e^{+} e^{-}, \mathrm{pp}\right)$ measured at collision energies below $100 \mathrm{GeV}$.
\end{abstract}

\section{Introduction}

In pp collisions at $\mathrm{TeV}$ center-of-mass energies, a significant disagreement [1] has been observed between the $p_{\mathrm{T}}$ spectra of measured $\Lambda$ and the spectra given by PYTHIA6 [2]. The study of the strange baryons and of their resonances in such collisions therefore provides an important reference for the tuning of the event generators.

Since $\Sigma^{0}$ and $\Lambda$ have different isospin values (respectively 1 and 0 ) but have the same quark content (uds) and similar masses $\left(m_{\Sigma^{0}}-m_{\Lambda} \sim 77 \mathrm{MeV}\right)$ [3], measuring their production and comparing them with PYTHIA model [2] could help to understand the strangeness production mechanisms, in particular those of baryons with non-zero isospin.

Finally, $\Sigma^{ \pm, 0}$ production measurements are important to quantify their feed-down contribution to the momentum spectra of their decay particles. The $\Sigma^{0}$ decays into $\Lambda+\gamma$ with $100 \%$ branching ratio, and it has a production rate of similar magnitude to the $\Lambda$ 's as reported in this article. Hence, $\Sigma^{0}$ induces a sizable feed-down on the $\Lambda[4]$ and also contributes to the contamination from decay photons to the low momentum part of the direct photon spectrum [5].

\section{Experimental setup and event selection}

A complete and detailed description of the ALICE detector and of its performance during the LHC Run 1 (2010-2013) can be found in [6]. We briefly outline only the sub-detectors

\footnotetext{
*e-mail: Alexander.Borissov@cern.ch
} 
utilized for the analysis reported here, namely the Inner Tracking System (ITS), the TimeProjection Chamber (TPC), the Photon Spectrometer (PHOS) and the V0 detectors.

Primary and secondary vertex reconstructions and charged-particle tracking are performed with the ITS and the TPC. They are located inside a solenoidal magnet providing a magnetic field of $0.5 \mathrm{~T}$ parallel to the LHC beam axis. The ITS is composed of six cylindrical layers of silicon detectors, located at radii between 3.9 and $43 \mathrm{~cm}$ from the nominal beam axis, covering a pseudo-rapidity range of $|\eta|<0.9$ and the full azimuth. The two innermost layers consist of Silicon Pixel Detectors (SPD) and provide trigger information. The spatial resolution of the ITS enables one to measure the distance of closest approach (DCA) to the primary vertex $(\mathrm{PV})$ of charged tracks with $p_{\mathrm{T}}>1 \mathrm{GeV} / c$ with a resolution of $75 \mu \mathrm{m}$ in the transverse plane. The TPC is a large cylindrical gaseous detector located between 85 and 250 $\mathrm{cm}$ from the beam axis and covering a pseudo-rapidity range of $|\eta|<0.9$ for tracks with full radial track length over the full azimuth. In addition, it provides particle identification via the measurement of the specific ionization energy loss $(\mathrm{d} E / \mathrm{dx})$ in the fill gas [6]. PHOS is made of $\mathrm{PbWO}_{4}$ crystals and has fine granularity with crystal size $2.2 \times 2.2 \times 18 \mathrm{~cm}^{3}$. It is located $4.6 \mathrm{~m}$ from the interaction point. PHOS subtends $260^{\circ}<\phi<320^{\circ}$ in azimuth and $|\eta|<0.13$ in pseudorapidity. The V0 detectors consist of two sets of scintillator tiles installed on both sides of the interaction point and covering $-3.7<\eta<-1.7$ and $2.8<\eta<5.1$. They are used for the interaction trigger of the experiment and for the rejection of beam-gas background interactions.

The data sample analyzed in this paper was recorded in 2010 during the LHC pp run at $\sqrt{s}=7 \mathrm{TeV}$ using a minimum-bias $\left(\mathrm{MB}_{\mathrm{OR}}\right)$ trigger [7]. The trigger required a single hit in either the SPD detector or in one of the two V0 counters, corresponding to the requirement of having at least one charged particle anywhere in the phase space covered by the SPD and the V0 detectors, which accounts for more than 8 units of pseudo-rapidity. A coincidence with the signals from the two beam pick-up (V0) counters located on each side of the interaction region, indicating the passage of colliding proton bunches, was also required. The contamination from beam-induced background was reduced to a negligible level with the help of the timing information of the V0 counters and by a selection on the correlation between clusters and tracklets reconstructed in the SPD, as discussed in detail in [6]. Events used for the data analysis are further required to have only one reconstructed primary vertex. A fraction of about $1.9 \%$ of the events are found to be pile-up events containing more than one distinct vertex and are discarded [7]. The residual pile-up after this selection is negligible.

Finally, events are selected for analysis by requiring that the position of the reconstructed primary vertex along the beam line lies within $\pm 10 \mathrm{~cm}$ from the center of the ALICE detector. A total amount of 458 million $\mathrm{MB}_{\mathrm{OR}}$ events $\left(\int L \mathrm{~d} t=4.34 \mathrm{nb}^{-1}\right)$ has been utilized for the analysis [7].

\section{Data analysis}

The $\Sigma^{0}$ baryon is reconstructed via invariant-mass analysis of its decays products in the prevalent (almost $100 \%$ branching fraction) electromagnetic decay channel $\Sigma^{0}\left(\bar{\Sigma}^{0}\right) \rightarrow \Lambda(\bar{\Lambda})+$ $\gamma$ [3]. Owing to its short lifetime, the decay vertex of the $\Sigma^{0}$ cannot be distinguished from the primary interaction vertex and the decay daughters appear as being emitted like primary particles. The identification of the $\Lambda(\bar{\Lambda})$ and of the photon daughters of the $\Sigma^{0}$ decay is performed thanks to the ALICE capabilities in reconstructing the secondary vertices, namely by reconstructing the weak-decay vertex $\mathrm{V}_{\Lambda}$ of $\Lambda \rightarrow \pi+p$, and the $e^{+} e^{-}$conversion vertex $\mathrm{V}_{\gamma}$ of the photon interaction with the detector material.

The main feature of $\Sigma^{0}$ decay is the low energy $(\sim 300 \mathrm{MeV})$ of the emitted photon due to the small mass difference between $\Sigma^{0}$ and $\Lambda$. The Photon Conversion Method (PCM) in the 
central tracking system was used for photon identification by employing the ITS and the TPC $[5,6]$. The secondary vertices $\left(\mathrm{VO}_{\gamma}\right)$ of the photon conversion to $e^{+} e^{-}$pairs generated in the inner detector material of ALICE are obtained during the data reconstruction by employing the full track capability of ITS and TPC. For the ALICE detector the probability of photon conversion in the central tracking system is about 0.089 and the reconstruction efficiency is about 0.67 [8], at maximum 0.73 for $p_{\mathrm{T}} \sim 3 \mathrm{GeV} / \mathrm{c}$ [6] with a stepeer decrease at low pT. The reliability of the method is based on the good agreement of the actual material budget and the simulated one [6].

To increase the $\Sigma^{0}$ data set, more relaxed selection criteria were used for gamma and Lambda selection with respect to the ones reported in Refs [6]. The tracks for $e^{-}\left(e^{+}\right)$candidates were selected if: (i) $|\eta|<0.9$, (ii) $p_{\mathrm{T}}>0.05 \mathrm{GeV} / c$, (iii) DCA between each track and $\mathrm{PV}$ is larger than $0.05 \mathrm{~cm}$, (iv) the ratio of the number of reconstructed to findable (maximum) TPC clusters $\left(N_{\text {ClusterTPC }} / N_{\text {FindClust }}\right)$ is larger than 0.35 . The measured energy loss in the TPC detector for $e^{-}\left(e^{+}\right)$candidate tracks are requested to be consistent with the expected energy loss $(\mathrm{d} E / \mathrm{d} x)$ within $-6 \sigma_{e}$ and $+7 \sigma_{e}$, where $\sigma_{e}$ is the standard deviation of the measured $\mathrm{d} E / \mathrm{d} x$ distribution for electrons in the TPC. In addition, in order to reduce pion and kaon contaminations for $p_{\mathrm{T}}<0.5 \mathrm{GeV} / c$, only the tracks with $|\mathrm{d} E / \mathrm{d} x|>1 \sigma_{\pi, K}$ are selected, where $\sigma_{\pi, K}$ are the standard deviations of measured $\mathrm{d} E / \mathrm{d} x$ distribution for pions $\left(\sigma_{\pi}\right)$ and kaons $\left(\sigma_{K}\right)$, respectively. To reduce pion and kaon contamination, a rejection of tracks with energy losses closer to the pion (kaon) line than one standard deviation of the measured $\mathrm{d} E / \mathrm{d} x$ distributions for pions $\left(\sigma_{\pi}\right)$ and kaons $\left(\sigma_{K}\right)$ was applied.

$\mathrm{V}_{\gamma}$ secondary vertex is identified if the DCA between the positive and negative track is lower than $1.5 \mathrm{~cm}$. To select photons among all secondary vertices, further selections were performed on the quality of the reconstructed $\mathrm{V}_{\gamma}$ [5]. In particular, a triangular twodimensional selection on the reduced $\chi^{2}$ of the photon conversion fit $\left(\chi_{\text {red }}^{2}\right)$ and the angle $\left(\psi_{\text {pair }}\right)$ between the plane perpendicular to the magnetic field of the ALICE magnet and the $e^{+} e^{-}$pair plane was introduced: $\left|\psi_{\text {pair }}\right|<\psi_{\text {pair,max }}\left(1-\chi_{\text {red }}^{2} / \chi_{\text {red,max }}^{2}\right)$ with $\psi_{\text {pair,max }}=0.1$ $\operatorname{rad}$ and $\chi_{\text {red,max }}^{2}=30$ [5]. A restriction on the distance $\left(R_{\gamma}\right)$ of the secondary vertices with respect to the nominal center of the detector $\left(5<R_{\gamma}<180 \mathrm{~cm}\right)$ is important to reduce the contamination from Dalitz decays.

An additional selection based on $q_{\mathrm{T}}=p_{e} \cdot \sin \theta_{\gamma, e}<0.06 \mathrm{GeV} / \mathrm{c}$, where $p_{e}$ is the electron momentum and $\theta_{\gamma, e}$ is the angle between gamma momentum $\left(p_{\gamma}\right)$ and electron momentum [9], was applied to increase the purity of the $\gamma$ vertices. Finally, only photons with $p_{\mathrm{T}}>0.02 \mathrm{GeV} / \mathrm{c}$ and $|\eta|<0.9$ were accepted.

Selection-criteria of the secondary vertex $\left(\mathrm{V}_{\Lambda}\right)$ described in Ref. [6] exploit the weak decay topology of $\Lambda(\bar{\Lambda}) \rightarrow \mathrm{p} \pi^{-}\left(\overline{\mathrm{p}} \pi^{+}\right)$with branching ratio of $63.9 \%$. Tracks from the weakdecay vertex $\left(\mathrm{V} 0_{\Lambda}\right) \Lambda\left(\bar{\Lambda} \rightarrow \mathrm{p} \pi^{-}\left(\pi^{+}\right)\right)$are requested to have opposite charge and the DCA with respect to the PV larger than $0.06 \mathrm{~cm}$. The DCA between $\mathrm{p}$ and $\pi^{-}$is requested to be smaller than $1.5 \mathrm{~cm}$. A further selection is applied by requesting the cosine of the pointing angle (the angle formed between the the $\mathrm{V}_{\Lambda}$ momentum and the flight trajectory) to be larger than 0.993, and the transverse distance between $\mathrm{V}_{\Lambda}$ and $\mathrm{PV}$ to be between 0.5 and $180 \mathrm{~cm}$.

A selection was applied in the Armenteros-Podolanski plane [9] $q_{\mathrm{T}, \Lambda}$ versus $\alpha_{\Lambda}$, where $q_{\mathrm{T}, \Lambda}$ is the relative transverse momentum of the proton with respect to the Lambda momentum and $\alpha_{\Lambda}=\frac{p_{l}^{p}-p_{l}^{\pi}}{p_{l}^{p}+p_{l}^{\pi}}$, where $p_{l}$ is the longitudinal momentum of $\mathrm{p}$ or $\pi$ with respect to the $\Lambda$ momentum direction. Two intervals $\left(0.2<\alpha_{\Lambda}<0.9\right.$ and $\left.-0.9<\alpha_{\Lambda}<-0.2\right)$ were used to select $\Lambda$ and $\bar{\Lambda}$, while a common range $\left(0.01<q_{\mathrm{T}, \Lambda}<0.17\right)$ was used. Finally, the invariant mass $M_{\mathrm{p} \pi}$ was requested to be within the interval of $1.110<M_{\mathrm{p} \pi}<1.120 \mathrm{GeV} / c^{2}$.

Furthermore the $\Sigma^{0}\left(\bar{\Sigma}^{0}\right)$ rapidity range $|y|<0.5$ is selected for the comparison with published $\Lambda p_{\mathrm{T}}$ spectrum [10]. 

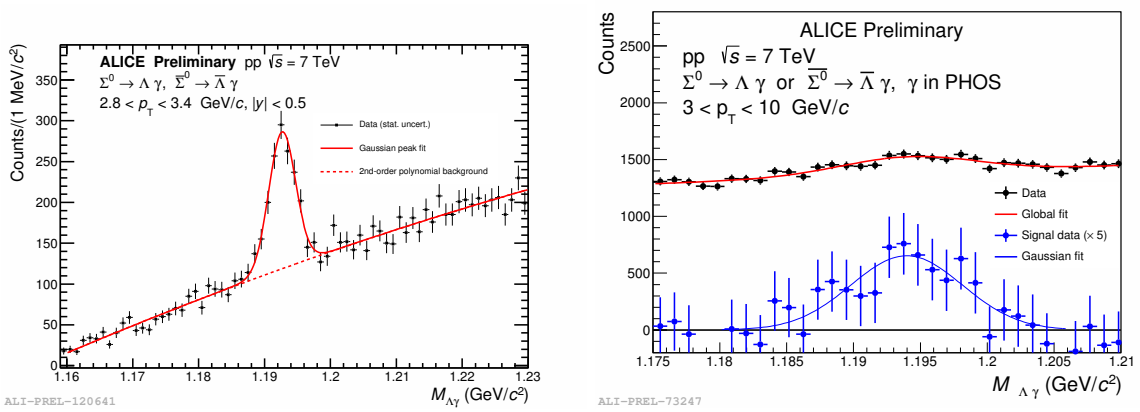

Figure 1. Invariant mass of $\Lambda \gamma$ and $\bar{\Lambda} \gamma$ pairs, where the $\Lambda$ or $\bar{\Lambda}$ is detected in the central tracking system and the photon is detected using the conversion method (left) or PHOS (right). The red curves are fits to the data using Gaussian peaks plus third-order polynomials to describe the background. The invariant mass distribution after the background subtraction is presented on the bottom part of the right plot.

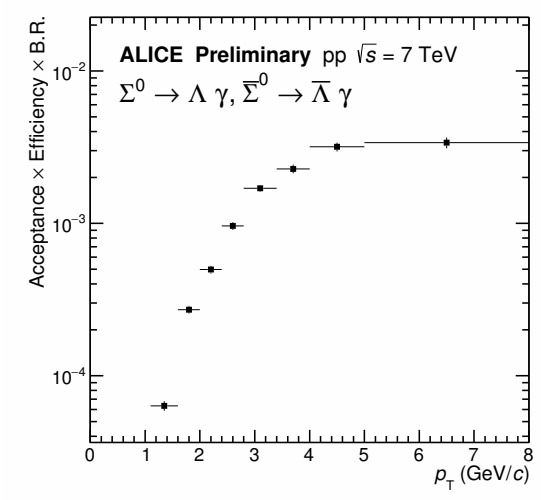

Figure 2. Acceptance $\times$ efficiency $\times$ B.R. correction for $\Sigma^{0}+\bar{\Sigma}^{0}$ in $|y|<0.5$. Only statistical uncertainties are shown.

The combined $\Sigma^{0}+\bar{\Sigma}^{0}$ signals are presented in Fig. 1 for the two independent methods of photon detection: PCM (left) and photon detection in PHOS (right). The peaks are fitted using a Gaussian peak added to a polynomial that describes the background. These fits have $\chi^{2} / n d f$ approximately equal to 1 .

The data from the electromagnetic calorimeter PHOS [6] for photon detection were also used for the observation of $\Sigma^{0}$ as a proof-of-principle due to the smaller acceptance and worse energy resolution: $\Delta E \sim 3 \% / \sqrt{E_{\gamma}(\mathrm{GeV})} \oplus 1.1 \%$. The invariant mass distribution of $\Sigma^{0}$ hyperons with a photon detected in PHOS, is presented in the right panel of Fig. 1.

The good energy resolution of the PCM [6] allows $\Sigma^{0}$ invariant mass peak to be reconstructed in the $p_{\mathrm{T}}$ region from 1.1 up to $8 \mathrm{GeV} / \mathrm{c}$, as can be seen in the left panel of Fig. 1. There is good agreement between the mean value of the $\Sigma^{0}$ mass and the vacuum value presented as a solid line. Finally, the PCM method was used for the reconstruction of $\Sigma^{0} p_{\mathrm{T}}$ spectrum. 


\subsection{Yield extraction}

The raw yields of $\Sigma^{0}+\bar{\Sigma}^{0}$ are extracted from fits to the invariant-mass distributions for $1.1<$ $p_{\mathrm{T}}<8 \mathrm{GeV} / c$. The $\Sigma^{0}$ signal is parametrised by a gaussian function, whereas the background contribution is parametrised by a third-order polynomial. The raw yield is obtained by integrating the Gaussian fit function in the region of \pm 3 standard deviations in each $p_{\mathrm{T}}$ bin. The statistical uncertainties on the raw yields are in the range 3-6\%.

The corrected $p_{\mathrm{T}}$ yield of $\Sigma^{0}$ is estimated as

$$
\frac{\mathrm{d}^{2} N}{\mathrm{~d} p_{\mathrm{T}} \mathrm{d} y}=\frac{1}{N_{I N E L}} \frac{N_{\text {raw }}\left(p_{\mathrm{T}}\right)}{\Delta y \Delta p_{\mathrm{T}}} \frac{1}{A \times \epsilon \times \mathrm{B} . \mathrm{R} .},
$$

where $N_{\text {raw }}\left(p_{\mathrm{T}}\right)$ is the measured $\Sigma^{0}+\bar{\Sigma}^{0}$ raw yield in the given $p_{\mathrm{T}}-$ bin, $\Delta p_{\mathrm{T}}$ and $\Delta y$ are the width of the corresponding $p_{\mathrm{T}}$ bin and the rapidity range $(|y|<0.5, \Delta \mathrm{y}=1)$, respectively. $\mathrm{A} \times \epsilon \times \mathrm{B} . \mathrm{R}$. is the correction factor that, in a given $p_{\mathrm{T}}$ bin, takes into account the geometrical acceptance (A), the $\Sigma^{0}$ detection efficiency $(\epsilon)$ and the fraction of $\Lambda$ in the decay channel $\mathrm{p} \pi^{-}$(B.R.) [3]. The corrected $p_{\mathrm{T}}$ distribution is normalized by the number of inelastic collisions $\left(N_{I N E L}\right)$, which is given by the number of the analyzed events corrected for the trigger efficiency with a factor $0.852_{-0.030}^{+0.062}$ [7].

The correction factors have been computed by using a detailed GEANT3 simulation of the ALICE detector. $\Sigma^{0}$ from inelastic pp interactions have been generated using PYTHIA6 Monte Carlo event generator with the Perugia-2011 tune [2]. The distribution of the $\mathrm{A} \times \epsilon \times \mathrm{B}$.R. is shown as a function of $p_{\mathrm{T}}$ in Fig. 2. Note that small values of correction factors at low $p_{\mathrm{T}}$ are mainly caused by the small conversion probability and reconstruction efficiency of low energy photons [6].

\subsection{Systematic uncertainties}

The total $\Sigma^{0}$ systematic uncertainty is determined by four independent sources of uncertainty: $\gamma$ and $\Lambda(\bar{\Lambda})$ selection, $\Sigma^{0}+\bar{\Sigma}^{0}$ raw yield extraction and the limited knowledge of material budget for $\Lambda$ and photon detection. The uncertainties $\left(\sigma_{m a t, \Lambda}, \sigma_{m a t, \gamma}\right)$ on $\Lambda$ and $\gamma$ related to the material budget are correlated. Then the total systematic uncertainty is evaluated as:

$$
\sigma_{s y s t}=\sqrt{\sigma_{\gamma}^{2}+\sigma_{\Lambda}^{2}+\sigma_{\Sigma^{0}}^{2}+\left(\sigma_{\mathrm{mat}, \Lambda}+\sigma_{\mathrm{mat}, \gamma}\right)^{2}},
$$

where $\sigma_{\gamma}$ and $\sigma_{\Lambda}$ are the relative systematic uncertainties for photons and $\Lambda(\bar{\Lambda})$ selection, $\sigma_{\Sigma^{0}}$ is the systematic uncertainty related to the $\Sigma^{0}+\bar{\Sigma}^{0}$ raw yield extraction. Because of the independent selection of $\Lambda$ and $\gamma$, only the uncertainties connected with $\Lambda$ and $\gamma$ selecton, correspondingly $\sigma_{\Lambda}$ and $\sigma_{\gamma}$ are uncorrelated. Linearly added material budget uncertainties $(8.5 \%)$ represent the largest contribution. The total uncertainty varies from 14.1 to $11.6 \%$ as $p_{\mathrm{T}}$ increases.

\section{Results and discussion}

The corrected yield of $\left(\Sigma^{0}+\bar{\Sigma}^{0}\right) / 2$ at mid-rapidity as a function of $p_{\mathrm{T}}$ in inelastic pp collisions at $\sqrt{s}=7 \mathrm{TeV}$ is shown in Fig. 3. The measurements span the $p_{\mathrm{T}}$ range from 1.1 to 8 $\mathrm{GeV} / c$. It was checked that the spectra obtained separately for $\Sigma^{0}$ and $\bar{\Sigma}^{0}$ are equal within the uncertainties. 


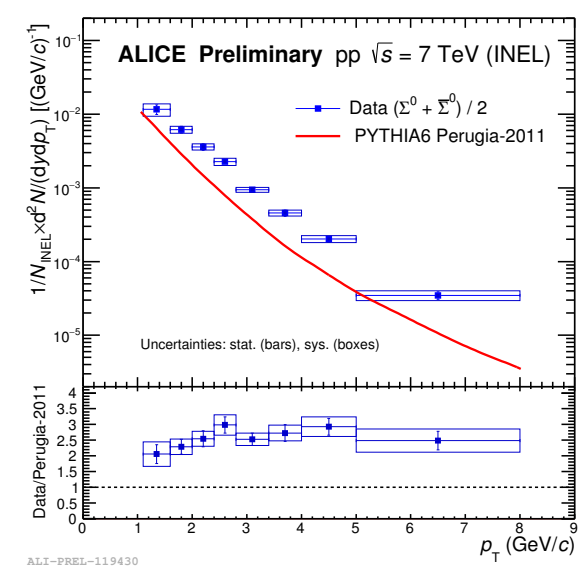

Figure 3. Transverse momentum spectrum of $\left(\Sigma^{0}+\bar{\Sigma}^{0}\right) / 2$ in the rapidity range $|y|<0.5$ is in the top panel. Statistical (bars) and systematic (boxes) uncertainties are included. The solid line represents the spectrum from PYTHIA6 Perugia-2011 [3]. Ratio of $\left(\Sigma^{0}+\bar{\Sigma}^{0}\right) / 2$ yield to the simulated one integrated in the corresponding $p_{\mathrm{T}}$ bins is in the bottom panel.

To estimate the $p_{\mathrm{T}}$-integrated yield and the mean transverse momentum of $\Sigma^{0}\left(\bar{\Sigma}^{0}\right)$, the spectra are fitted with a Lévy-Tsallis function [11],

$$
\frac{\mathrm{d}^{2} N}{\mathrm{~d} p_{\mathrm{T}} \mathrm{d} y}=p_{\mathrm{T}} \frac{\mathrm{d} N}{\mathrm{~d} y} \frac{(n-1)(n-2)}{n C\left[n C+M_{\Sigma^{0}}(n-2)\right]}\left[1+\frac{\sqrt{p_{\mathrm{T}}^{2}+M_{\Sigma^{0}}^{2}}-M_{\Sigma^{0}}}{n C}\right]^{-n},
$$

where $M_{\Sigma^{0}}$ is the PDG value of $\Sigma^{0}$ mass [3]. The free parameters of the inverse slope $C$, the exponent $n$ and $\mathrm{d} N / \mathrm{d} y$, which represents the particle yield per unit rapidity are used to extrapolate the distribution at low and large $p_{\mathrm{T}}$. This fit function is widely used to describe all identified particle spectra in pp collisions [12].

The transverse momentum spectra of $\left(\Sigma^{0}+\bar{\Sigma}^{0}\right) / 2$ are compared to the results from PYTHIA6, Perugia-2011 tune [2], see Fig. 3. This generator significantly underestimates the yield in $p_{\mathrm{T}}$. In the bottom panel of Fig. 3, one can observe the ratio of data to the model, which is rather flat in $p_{\mathrm{T}}$ around a constant value $2.41 \pm 0.09$.

The differential yield ratio of $\left(\Sigma^{0}+\bar{\Sigma}^{0}\right) /(\Lambda+\bar{\Lambda})$ is presented as a function of $p_{\mathrm{T}}$ in the bottom panel of Fig. 4. Note the trend of the monotonic increase of the ratio for $p_{\mathrm{T}}>3$ $\mathrm{GeV} / \mathrm{c}$, whereas at low $p_{\mathrm{T}}$ the ratio is significantly suppressed relative to $\Lambda$. Presumably, the increase of the $\frac{\Sigma^{0}}{\Lambda}$ cross section ratio is caused by the quicker increase of the $\Sigma^{0}$ production cross section with $p_{\mathrm{T}}$ relative to $\Lambda$. At low $p_{\mathrm{T}}$, the $\Lambda$ spectrum contains $\Lambda$ from $\Sigma^{0}$ decays and the ratio accordingly decreases. The growth of the $\frac{\Sigma^{0}}{\Lambda}$ ratio with $p_{\mathrm{T}}$ also may be connected with the contribution of heavier resonance decays into the spectrum of the measured ground state baryons $\Sigma^{0}$ and $\Lambda$.

The integrated yield at mid-rapidity for $\Sigma^{0}\left(\bar{\Sigma}^{0}\right)$ is obtained by integrating the data over the measured range $\left(p_{\mathrm{T}}>1.1 \mathrm{GeV} / c\right)$, see Fig. 3, and using the Lévy-Tsallis fit to extrapolate the yield in the unmeasured range $\left(p_{\mathrm{T}}<1.1 \mathrm{GeV} / c\right)$. Note that the fraction of yield extracted from the fit-function in the unmeasured $p_{\mathrm{T}}$ region $\left(0<p_{\mathrm{T}}<1.1 \mathrm{GeV}\right)$, i.e. from the low- $p_{\mathrm{T}}$ extrapolation to the total yield is quite large and equal to 0.58 from the Lévy-Tsallis fit $(0.57$ 


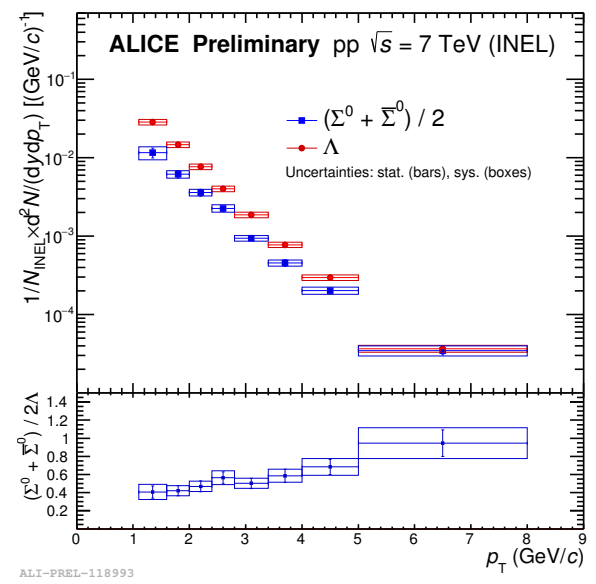

Figure 4. The differential yields of $\left(\Sigma^{0}+\bar{\Sigma}^{0}\right) / 2$ and $\Lambda$ [10] as a function of $p_{\mathrm{T}}$ are in the top panel. The differential yield ratios of $\left(\Sigma^{0}+\bar{\Sigma}^{0}\right) / 2$ to $\Lambda$ as a function of $p_{\mathrm{T}}$ are in the bottom panel.

from Boltzmann-Gibbs Blast-Wave fit [13]). The systematic uncertainty on the total yield due to this extrapolation was calculated by varying the fit functions (the $m_{T}$-exponential, $p_{\mathrm{T}}$-exponential, Fermi-Dirac, Boltzmann, Boltzmann-Gibbs Blast-Wave and Bose-Einstein fits [14]) and is included as an independent source of systematic uncertainty. The fraction of the yield due to the extrapolation to high- $p_{\mathrm{T}}$ is found to be negligible.

The integrated yield ratios of $\Sigma^{0}$ to $\Lambda$ from various collision systems at different energies are shown as a function of $\sqrt{s}$ in Fig. 5. While yields of $\Sigma^{0}$ have been measured in many different collision systems at low and intermediate energies, there exist only few results in high energy collisions including the ALICE result. The new ALICE data point from pp collisions at $7 \mathrm{TeV}$ is similar to the $\Sigma^{0} / \Lambda$ ratio measured in $\mathrm{e}^{+} \mathrm{e}^{-}$collisions at $100 \mathrm{GeV}$.

A similar value for the ratio $\left(\Sigma^{0} / \Lambda=0.27\right)$ was obtained using the statistical hadronization model [16]. The value of the ratio $\Sigma^{0} / \Lambda$ obtained at $\sqrt{s}=7 \mathrm{TeV}$ is is consistent with those obtained in $e^{+} e^{-}$collisions and proton-(anti)proton collisions in a wide energy range below $90 \mathrm{GeV}$. Ratios between different species should be energy independent [16] because of constant values of the hadronization temperature and strangeness suppression factor.

\section{Summary}

The $\Sigma^{0}\left(\bar{\Sigma}^{0}\right)$ hyperons produced in pp collisions at $\sqrt{s}=7 \mathrm{TeV}$ are successfully reconstructed via their electromagnetic decays to $\Lambda(\bar{\Lambda})$ and $\gamma$. The $p_{\mathrm{T}}$ distribution of $\left(\Sigma^{0}+\right.$ $\left.\bar{\Sigma}^{0}\right) / 2$ is obtained and compared with the results of simulations with the PYTHIA6 generator, which significantly underestimates the differential yields. Furthermore the differential yield ratios of $\Sigma^{0}$ to $\Lambda$ increase with $p_{\mathrm{T}}$. The $\left(\Sigma^{0}+\bar{\Sigma}^{0}\right) / 2 \Lambda$ ratio measured in high-energy pp collisions is consistent with the values measured in $e^{+} e^{-}$and pp collisions below $100 \mathrm{GeV}$ within uncertainties. The integrated yield ratio of $\Sigma^{0}$ to measured $\Lambda$ in pp collisions at $\sqrt{s}=7 \mathrm{TeV}$ is consistent with the prediction of statistical hadronization model [16]. The current measurement represents a relevant baseline for further investigation in pp collisions at different energies, as well as in $\mathrm{p}-\mathrm{Pb}$ and $\mathrm{Pb}-\mathrm{Pb}$ collisions. 


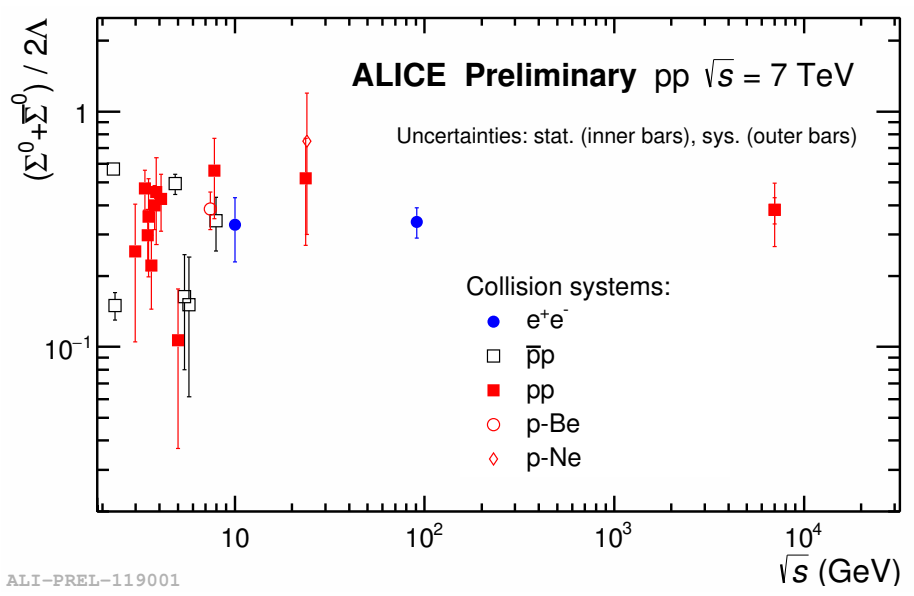

Figure 5. Energy dependence of the the integrated yield ratios $\Sigma^{0}+\bar{\Sigma}^{0}$ to $2 \Lambda$. Non-ALICE points are taken from Ref. [15].

\section{References}

[1] ATLAS Collaboration, Phys. Rev. D 85, 012001 (2012)

[2] P.Z. Skands, Phys. Rev. D 82, 074018 (2010)

[3] M. Tanabashi et. al., Phys. Rev. D 98, 030001 (2018)

[4] J. Lettessier and J. Rafelski, Hadrons and Quark-Gluon Plasma (Cambridge University Press, 2005)

[5] ALICE Collaboration, Phys. Lett. B 754, 235-248 (2016)

[6] ALICE Collaboration, Int. J. Mod. Phys. A 29, 1430044 (2014)

[7] ALICE Collaboration, Eur. Phys. J. C 73, 2456 (2013)

[8] ALICE Collaboration, Phys. Lett. B 717, 162-172 (2012)

[9] J. Podolanski and R. Armenteros, Philos. Mag. 45 (360), 13-30 (1954)

[10] D.D. Chinellato for ALICE collaboration, arXiv:1211.7298 (2012)

[11] C. Tsallis, J. Statist. Phys. 52, 479-487 (1988)

[12] ALICE Collaboration, Eur. Phys. J. C 75, 1 (2015)

[13] E. Schnedermann, J. Sollfrank, U.W. Heinz, Phys. Rev. C 48, 2462-2475 (1993)

[14] STAR Collaboration, Phys. Rev. C 79, 034909 (2009)

[15] G. Van Buren for the STAR Collaboration, J.Phys. G 31, 195 (2005)

[16] F. Becattini, P. Castorina, A. Milov and H. Satz, J. Phys. G 38, 025002 (2011) 